S Research S quare

\title{
Widened difference of incidence and survival between different races in epithelial ovarian cancer: a period analysis.
}

\section{Li Li ( $\sim$ Lili89650@163.com )}

People's Hospital of Xinjiang Uygur Autonomous Region https://orcid.org/0000-0003-3510-0530 Lili Han

People's Hospital of Xinjiang Uygur Autonomous Region

\section{Sulaiya Husaiyin}

People's Hospital of Xinjiang Uygur Autonomous Region

Miherinisha Maimaiti

People's Hospital of Xinjiang Uygur Autonomous Region

Mayinuer Niyazi

People's Hospital of Xinjiang Uygur Autonomous Region

\section{Research}

Keywords: Epithelial Ovarian cancer, Incidence, Relative survival, Period analysis, Socioeconomic status

Posted Date: April 2nd, 2020

DOI: https://doi.org/10.21203/rs.3.rs-20560/v1

License: (c) (1) This work is licensed under a Creative Commons Attribution 4.0 International License. Read Full License 


\section{Abstract}

Introduction To describe the incidence and relative survival in women with epithelial ovarian cancer (EOC) in a population-based cohort in the four decades after diagnosis. EOC is the major pathological type of all ovarian cancers, however, there is limited information on changes of long-term survival in EOC in the four decades.

Methods We extracted the incidence and relative survival data from Surveillance, Epidemiology, and End Results (SEER) registries to assess epidemiological changes of patients with EOC from 1974 to 2013. The survival disparities of patients with EOC among four decades, age, race, and socioeconomic status (SES) were performed by Kaplan-Meier curves.

Results The overall incidence of EOC gradually declined from 11.4 to 9.0 per 100,000 in the past four decades. The median survival increased from 27 months in the first decades to 48 months in the fourth decade, with 5-year relative survival rate (RSR) improving from $32.3 \%$ to $44.3 \%$ in the same period. However, the median survival differences increased from 11 months to 18 months between Whites and Blacks and increased from 7 months to 12 months between low-poverty group and high-poverty group respectively over the past four decades.

Discussion This study indicated that the incidence and RSR of EOC patients had improved in the past four decades. But the survival gap between different races and SES gradually widened. More importantly, this study will promote the improvement of health care system and clinical management to erase the survival differences in SES groups and races identified in this study, thereby optimize the clinical outcome.

\section{Background}

Ovarian cancer is the major lethal cause of women with gynecologic cancer, with an estimated 22,530 new cases being diagnosed and 13,980 death cases of ovarian cancer around the world in $2019^{1}$. Due to lack of specific symptoms and early detection methods, most patients with ovarian cancer had developed to advanced stage (Stage III and IV) when they were diagnosed, which have an unsatisfactory prognosis in spite of immediate and aggressive treatment ${ }^{2}$. The five-year survival of patients with advanced-stage ovarian cancer is approximately $30 \%$, with increased risk of metastasis and recurrence ${ }^{2}$. Although the treatment of cancer has been greatly improved, including chemotherapy, radiotherapy, targeted therapy and biological therapy, the survival haven't been found improved significantly, with 5-year survival rate below $45 \%$, indicating that an urgent need to understand the causes and analysis epidemiological trends of ovarian cancer, which could help improve clinical management and treatment outcomes.

Epithelial ovarian cancer (EOC) is the main pathological type of all ovarian cancers, accounting for approximately $90 \%{ }^{3}$. However, previous epidemiological information for EOC was limited, therefore, we performed a comprehensive population-based analysis to clarify the prognostic significance of age, race 
and socioeconomic status (SES) in the incidence and relative survival of EOC patients. Data in 19742013 from the US Surveillance, Epidemiology and End Results (SEER) cancer registry program, were used to assess the association of incidence and relative survival with age, race and SES.

Recently growing evidence showed that the health care system appeared increasingly distinct racial and SES differences in various aspects of the United States, which has been focused by health care politicians ${ }^{4-6}$. Thus, the aim of the study was not only to examine population-level changes over time in incidence and long-term relative survival for patients with EOC diagnosed among 1974-2013, but also to evaluate the relative survival differences between different race and SES in EOC.

\section{Methods}

\section{Data source}

All data on patients with primary invasive EOC were obtained from SEER program during 1974-2013. Data on incidence and relative survival rate, respectively, were extracted from the original nine and 18 SEER sites.

\section{Stratified Ovarian Cancer Cases Over The Past Three Decades}

We utilized SEER*Stat version 8.3.5 to obtain and analyze the incidence and relative survival data. Patient inclusion criteria were based on the ICD-0-3 (International Classification of Diseases for Oncology, 3rd edition) and WHO 2008 site code C56.97 from 1974 to 2013. We excluded EOC cases diagnosed by autopsy or reported only on a death certificate. In this study, variables included year of diagnosis, age, race and SES. Data on incidence and relative survival data between 1974 and 2013 were analyzed after being divided by four decades. According to age, the patients were divided into four groups $(0-44,45-54$, 55-69, 70 + years), and race was classified as White, Black, and Other (American Indian/Alaska Native, Asian/Pacific islander). The county poverty rate was used to define area SES, which was divided into three levels using the National Cancer Institute monograph: < 10\% (low-poverty areas), 10\%-19.99\% (medium-poverty areas), and $\geq 20 \%$ (high-poverty areas) ${ }^{8-11}$.

\section{Statistical analysis}

Incidence was expressed per 100,000 population and age-adjusted to 2000 U.S. standard population. The Kaplan-Meier log-rank test was performed to compare the survival differences between subgroups of each variable and $p$ value $<0.01$ was considered to be statistically significant.

\section{Results}




\section{Incidence trend of EOC between 1974 and 2013}

Total 53,269 patients with EOC were diagnosed among 1974-2013 from original 9 SEER registry sites. The overall incidence of EOC gradually decreased from 11.4 to 9.0 per 100,000 in the four decades. And all age groups also were found to present a similar downward incidence trend, with the most significant decline in the 0-44 age group, which decreased by $33.3 \%$, from 2.4 to 1.8 per 100,000 (Fig. 1a, 1b, Supplementary Table S1).

Over the past four decades, the incidence of EOC in three race subgroups had declined over time. A similar incidence of EOC was observed between Blacks and Others, with Whites having the highest incidence in the past four decades. However, as EOC incidence in Whites declined faster in the four decades, the incidence gaps among the three race subgroups become narrowing (Fig. 1c).

The incidence of EOC had declined in the three subgroups of SES, with the lowest incidence in the high poverty group over the past four decades (from 9.5 in 1974-1983 to 7.1 in 2004-2013). In the past four decades, the low poverty group showed the highest incidence of 11.8, 12.0, 10.7, 9.0 per 100,000 each decade. In addition, the incidence of medium poverty group was between low-poverty group and highpoverty group (Fig. 1e).

\section{Changes Of Relative Survival In Eoc During 1974-2013}

Totally 93,639 EOC cases were obtained from 18 SEER sites in the past four decades. There was an improvement in median survival of EOC patients (from 27 months in the first decades to 48 months in the fourth decade) as well as relative survival rate (RSR) and survival time for patients with EOC in the four decades (Table 1, Fig. 2a). The 5-year RSR in 1994-2003 was higher than that in 1984-1993 and 19741983 (41.3\% in $1994-2003$ vs $34.4 \%$ in $1984-1993$ vs $32.3 \%$ in $1974-1983, p<0.0001$ ), and even higher in $2004-2013$ ( $44.3 \%$ vs $41.1 \%, p<0.0001)$ with an significant increase by $37.5 \%$ compared to the first decade. The trend of survival improvement was also observed in the RSR of 1 year, 2 year and 10 year. Furthermore, improvement in survival of all age groups was demonstrated by Kaplan-Meier survival analysis with statistical significance over four decades ( $p<0.0001$; Fig. $2 b)$. 
Table 1

Relative survival rates of epithelial ovarian cancer patients during the periods of 1974-1983, 1984-1993, 1994-2003, and 2004-2013 at eighteen SEER sites. Data are mean \pm standard error of the mean, with number of patients in parentheses.

\begin{tabular}{|c|c|c|c|c|}
\hline \multirow{2}{*}{$\begin{array}{l}\text { Age } \\
\text { Group }\end{array}$} & \multicolumn{3}{|c|}{ Decade } & \multirow[b]{2}{*}{$2004-2013$} \\
\hline & $1974-1983$ & 1984-1993 & $1994-2003$ & \\
\hline \multicolumn{5}{|c|}{ 12-Mo RS } \\
\hline All & $\begin{array}{l}63.9 \pm \\
0.5(11521)\end{array}$ & $69.8 \pm 0.4(14260)^{\star \star \star}$ & $\begin{array}{l}74.7 \pm \\
0.3(27680) \star \star \star\end{array}$ & $\begin{array}{l}76.8 \pm \\
0.2(40178) \star \star \star\end{array}$ \\
\hline $0-44$ & $86.0 \pm 0.9(1379)$ & $90.2 \pm 0.7(1647)^{\star \star \star}$ & $89.2 \pm 0.6(2978)^{\star \star}$ & 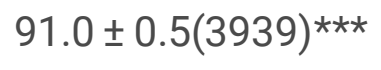 \\
\hline $45-54$ & $78.2 \pm 0.9(2194)$ & $85.5 \pm 0.8(2164)^{\star \star \star}$ & $88.6 \pm 0.4(5385)^{\star \star \star}$ & $89.0 \pm 0.4(8189)^{\star \star \star}$ \\
\hline $55-69$ & $65.3 \pm 0.7(4690)$ & $75.1 \pm 0.6(5377)^{\star \star \star}$ & $81.1 \pm 0.4(9226)^{\star \star \star}$ & $\begin{array}{l}83.2 \pm \\
0.3(14848) \star \star \star\end{array}$ \\
\hline $70+$ & $42.6 \pm 0.9(3258)$ & $50.6 \pm 0.7(5075)^{\star \star \star \star}$ & $\begin{array}{l}57.0 \pm \\
0.5(10091) \star \star \star\end{array}$ & $\begin{array}{l}57.5 \pm \\
0.5(13202) \star \star \star\end{array}$ \\
\hline \multicolumn{5}{|c|}{ 24-Mo RS } \\
\hline All & $46.6 \pm 0.5$ & $53.2 \pm 0.4^{\star \star \star}$ & $62.4 \pm 0.3^{\star \star \star}$ & $65.7 \pm 0.3^{\star \star \star}$ \\
\hline $0-44$ & $75.1 \pm 1.2$ & $80.1 \pm 1.0 * \star \star$ & 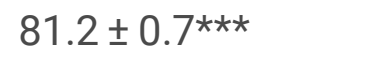 & $84.9 \pm 0.6 * \star \star$ \\
\hline $45-54$ & $58.7 \pm 1.1$ & $70.1 \pm 1.0 * \star \star$ & $77.4 \pm 0.6^{\star \star \star}$ & $80.2 \pm 0.5^{\star \star \star}$ \\
\hline $55-69$ & $44.5 \pm 0.7$ & $54.9 \pm 0.7 * \star \star$ & $67.4 \pm 0.5^{\star \star \star}$ & $71.1 \pm 0.4^{\star \star \star}$ \\
\hline $70+$ & $29.0 \pm 0.9$ & $35.1 \pm 0.7 * \star \star *$ & $43.8 \pm 0.5^{\star \star \star}$ & $44.7 \pm 0.5^{\star \star \star}$ \\
\hline \multicolumn{5}{|c|}{ 60-Mo RS } \\
\hline All & $32.3 \pm 0.5$ & $34.4 \pm 0.4^{\star \star \star}$ & $41.1 \pm 0.3^{\star \star \star}$ & $44.3 \pm 0.3^{\star \star \star}$ \\
\hline $0-44$ & $62.1 \pm 1.3$ & $63.4 \pm 1.2$ & $64.0 \pm 0.9$ & 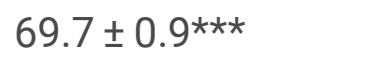 \\
\hline $45-54$ & $41.7 \pm 1.1$ & $49.2 \pm 1.1^{\star \star \star}$ & $55.3 \pm 0.7^{\star \star \star}$ & 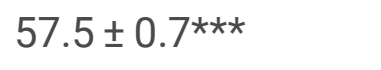 \\
\hline $55-69$ & $28.3 \pm 0.7$ & 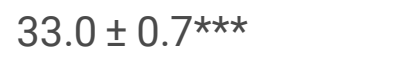 & $42.3 \pm 0.5^{\star \star \star}$ & $46.1 \pm 0.5^{\star \star \star}$ \\
\hline $70+$ & $18.2 \pm 0.8$ & $19.5 \pm 0.6$ & $24.9 \pm 0.5^{\star \star \star}$ & $25.8 \pm 0.5^{\star \star \star}$ \\
\hline \multicolumn{5}{|c|}{$\begin{array}{l}\text { 120-Mo } \\
\text { RS }\end{array}$} \\
\hline All & $27.0 \pm 0.5$ & $27.4 \pm 0.4$ & $30.8 \pm 0.3^{\star \star \star}$ & $34.3 \pm 0.4^{\star \star \star}$ \\
\hline
\end{tabular}

Abbreviations: Mo, month; RS, relative survival; SEM, standard error of the mean.

${ }^{*} p<0.01,{ }^{* \star} p<0.001$, and ${ }^{* *} p<0.0001$ for comparisons with the preceding decade. 


\begin{tabular}{|c|c|c|c|c|}
\hline Age & & Decade & & \\
\hline $0-44$ & $56.1 \pm 1.4$ & $56.4 \pm 1.2$ & $57.0 \pm 0.9$ & $60.0 \pm 1.2^{\star \star \star}$ \\
\hline $45-54$ & $35.6 \pm 1.1$ & $39.7 \pm 1.1^{\star \star \star}$ & $43.0 \pm 0.7^{\star \star \star *}$ & 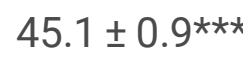 \\
\hline $55-69$ & $22.2 \pm 0.7$ & $33.0 \pm 0.7 * \star \star$ & $30.2 \pm 0.5^{\star \star \star}$ & 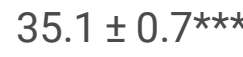 \\
\hline $70+$ & $14.3 \pm 0.9$ & $15.0 \pm 0.7$ & $16.9 \pm 0.5^{\star}$ & 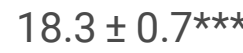 \\
\hline \multicolumn{5}{|c|}{ Abbreviations: Mo, month; RS, relative survival; SEM, standard error of the mean. } \\
\hline
\end{tabular}

Additionally, the survival of all race subgroups improved, with the 5-year RSR of Whites the most significant improvement, increasing by $40.56 \%$, from 31.8 to 44.7 over four decades. In the four decades, the highest survival rate was seen in Others, and Whites showed higher survival compared to Blacks. The 5-year RSR survival difference between Whites and Black widened in 1984-1993 (34.3\% vs 28.4\%) and greatly become widener in the last two decades (41.3\% vs $30.8 \%$ in $1994-2003$ and $44.7 \%$ vs 30.9 in 2004-2013). A similar tendency of survival difference was also observed over time in 10-year RSRs (Fig. 3a, Table 2). 
Table 2

12-month, 60-month and 120-month relative survival rates of epithelial ovarian cancer patients according to race, age group, and calendar period from 1974 to 2013 at eighteen

SEER sites. Data are means \pm standard error of the mean, with number of patients in parentheses.

\begin{tabular}{|c|c|c|c|c|}
\hline \multicolumn{5}{|c|}{ Race } \\
\hline Decade & Age Group & White & Black & Other \\
\hline \multirow[t]{18}{*}{$74-83$} & 12-Mo RS & & & \\
\hline & All & $64.1 \pm 0.5(10546)$ & $56.3 \pm 2.1(598)^{\star \star \star}$ & $70.7 \pm 2.4(377)^{\star}$ \\
\hline & $0-44$ & $86.3 \pm 1.0(1207)$ & $82.1 \pm 3.8(105)$ & $86.7 \pm 4.2(67)$ \\
\hline & $45-54$ & $78.9 \pm 0.9$ (1982) & $63.2 \pm 4.7(109)^{\star \star \star}$ & $80.7 \pm 3.9(103)$ \\
\hline & $55-69$ & $65.7 \pm 0.7(4301)$ & $56.2 \pm 3.3(243)^{\star \star}$ & $70.2 \pm 3.8(146)$ \\
\hline & $70+$ & $43.2 \pm 0.9(3056)$ & $31.6 \pm 4.1(141)^{\star \star}$ & $37.1 \pm 6.3(61)$ \\
\hline & 60-Mo RS & & & \\
\hline & All & $31.8 \pm 0.5$ & $34.0 \pm 2.1$ & $40.5 \pm 2.6^{\star \star *}$ \\
\hline & $0-44$ & $60.8 \pm 1.4$ & $74.1 \pm 4.4^{\star \star \star}$ & $65.8 \pm 5.9$ \\
\hline & $45-54$ & $42.0 \pm 1.1$ & $32.0 \pm 4.6 * \star \star$ & $46.8 \pm 5.0$ \\
\hline & $55-69$ & $28.2 \pm 0.7$ & $28.7 \pm 3.1$ & $32.9 \pm 4.0$ \\
\hline & $70+$ & $18.4 \pm 0.8$ & $12.7 \pm 0.8$ & $19.5 \pm 3.4$ \\
\hline & 120-Mo RS & & & \\
\hline & All & $26.7 \pm 0.5$ & $27.1 \pm 2.0$ & $34.0 \pm 2.6^{\star *}$ \\
\hline & $0-44$ & $54.7 \pm 1.5$ & $69.3 \pm 4.7^{\star \star \star *}$ & $60.2 \pm 6.1$ \\
\hline & $45-54$ & $35.9 \pm 1.1$ & $23.7 \pm 4.4^{\star \star \star}$ & $41.7 \pm 5.0 * *$ \\
\hline & $55-69$ & $22.3 \pm 0.7$ & $19.3 \pm 2.9$ & $22.6 \pm 3.7$ \\
\hline & $70+$ & $15.2 \pm 0.9$ & $8.3 \pm 3.3^{\star \star \star}$ & $18.5 \pm 6.6$ \\
\hline \multirow[t]{3}{*}{$84-93$} & 12-Mo RS & & & \\
\hline & All & $70.0 \pm 0.4(12729)$ & $60.5 \pm 1.8(768)^{\star \star \star}$ & $76.0 \pm 1.6(763)^{\star \star}$ \\
\hline & $0-44$ & $90.3 \pm 0.8(1403)$ & $92.0 \pm 2.8(98)$ & $87.7 \pm 2.7(146)$ \\
\hline
\end{tabular}

Abbreviations: Mo, month; RS, relative survival; SEM, standard error of the mean.

${ }^{*} p<0.01,{ }^{* *} p<0.001$, and ${ }^{* *} \mathrm{p} p 0.0001$ for comparisons with the White group. 


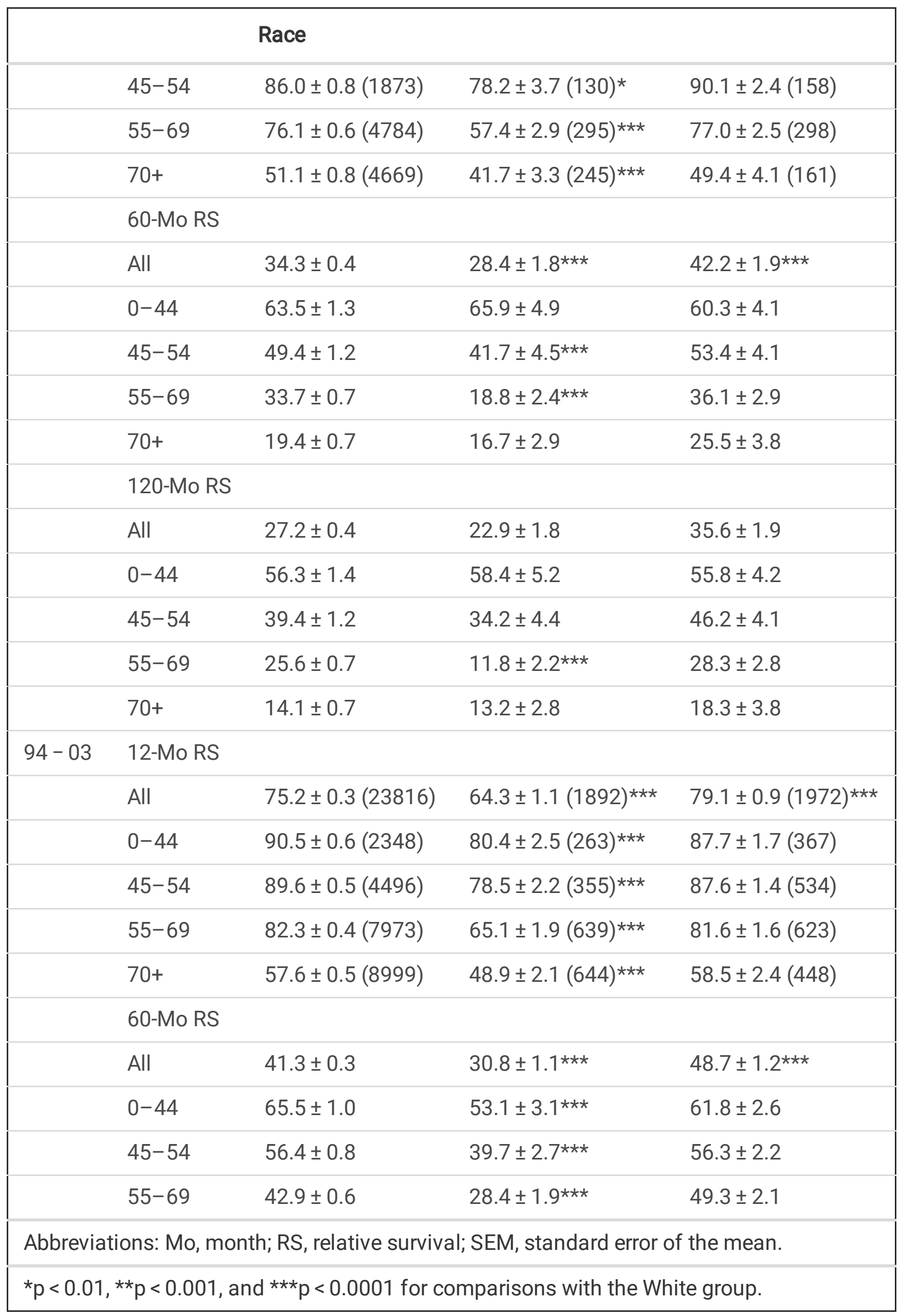




\begin{tabular}{|c|c|c|c|c|}
\hline & & Race & & \\
\hline & $70+$ & $25.2 \pm 0.5$ & $18.1 \pm 1.8^{\star \star \star}$ & $27.6 \pm 2.3^{\star \star \star}$ \\
\hline \multicolumn{5}{|c|}{ 120-Mo RS } \\
\hline & All & $30.7 \pm 0.3$ & $23.1 \pm 1.1^{\star \star \star}$ & $39.4 \pm 1.2^{\star \star \star}$ \\
\hline & $0-44$ & $54.6 \pm 1.1$ & $44.2 \pm 3.2^{\star \star \star}$ & $54.5 \pm 2.7$ \\
\hline & $45-54$ & $43.8 \pm 0.8$ & $29.1 \pm 2.6^{\star \star \star}$ & $46.2 \pm 2.2$ \\
\hline & $55-69$ & $30.4 \pm 0.6$ & $19.9 \pm 1.8^{\star \star \star}$ & $37.9 \pm 2.1^{\star \star \star}$ \\
\hline & $70+$ & $17.0 \pm 0.6$ & $12.8 \pm 1.9 * \star \star$ & $20.0 \pm 2.3$ \\
\hline $04-13$ & \multicolumn{4}{|l|}{ 12-Mo RS } \\
\hline & All & $77.3 \pm 0.2(33636)$ & $65.5 \pm 0.9(3141)^{\star \star \star}$ & $82.0 \pm 0.7(3401)^{\star \star \star}$ \\
\hline & $0-44$ & $92.3 \pm 0.5(3061)$ & 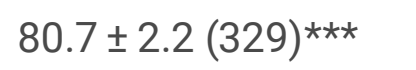 & $89.8 \pm 1.3(549)$ \\
\hline & $45-54$ & $89.5 \pm 0.4(6569)$ & $79.1 \pm 1.7(622)^{\star \star \star}$ & $91.9 \pm 0.9(998)$ \\
\hline & $55-69$ & $84.4 \pm 0.3(12501)$ & $68.8 \pm 1.4(1217)^{\star \star \star}$ & $85.2 \pm 1.1(1130)$ \\
\hline & $70+$ & $58.4 \pm 0.5(11505)$ & $47.4 \pm 1.7(973)^{\star \star \star}$ & $57.6 \pm 1.9(724)$ \\
\hline \multicolumn{5}{|c|}{ 60-Mo RS } \\
\hline & All & $44.7 \pm 0.3$ & $30.9 \pm 1.1^{\star \star \star}$ & $52.8 \pm 1.1^{\star \star \star}$ \\
\hline & $0-44$ & $71.1 \pm 1.0$ & $53.1 \pm 3.3 * \star \star$ & $72.2 \pm 2.3$ \\
\hline & $45-54$ & $58.6 \pm 0.7$ & $37.7 \pm 2.5^{\star \star \star}$ & $62.6 \pm 1.9$ \\
\hline & $55-69$ & $47.0 \pm 0.6$ & $31.0 \pm 1.7^{\star \star \star}$ & 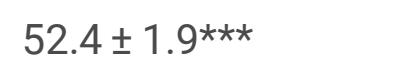 \\
\hline & $70+$ & $26.6 \pm 0.6$ & $17.7 \pm 1.7^{\star \star \star}$ & $23.8 \pm 2.1$ \\
\hline \multicolumn{5}{|c|}{ 120-Mo RS } \\
\hline & All & $35.2 \pm 0.5$ & $22.5 \pm 1.3^{\star \star \star}$ & $41.3 \pm 1.5^{\star \star \star}$ \\
\hline & $0-44$ & $60.5 \pm 1.4$ & $45.8 \pm 3.6 * \star \star$ & $60.6 \pm 4.0$ \\
\hline & $45-54$ & $45.8 \pm 1.0$ & 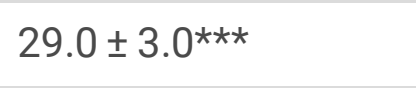 & $51.0 \pm 2.9 \star \star \star$ \\
\hline & $55-69$ & $35.5 \pm 0.8$ & $22.1 \pm 2.3^{\star \star \star}$ & $40.5 \pm 2.5^{\star \star \star}$ \\
\hline & $70+$ & $19.2 \pm 0.8$ & $10.0 \pm 2.2^{\star \star \star}$ & $16.2 \pm 3.0$ \\
\hline \multicolumn{5}{|c|}{ Abbreviations: Mo, month; RS, relative survival; SEM, standard error of the mean. } \\
\hline${ }^{*} \mathrm{p}<0.0$ & $<0.001$ & $\star \star * \mathrm{p}<0.0001 \mathrm{fo}$ & arisons with the & e group. \\
\hline
\end{tabular}


When survival time of SES subgroups was analyzed, it was showed that the RSR of low-poverty group was the highest while that of high-poverty group was the lowest. Over the past four decades, the survival time of all SES subgroups had improved, but over time, the survival gaps between low-poverty group and high-poverty group become widener and widener. For example, in 1984-1993, the 5-year RSR of lowpoverty group and high-poverty group was $35.1 \%$ vs $32.5 \%$ respectively, and the gap between 1994-2003 and 2004-2013 increased to $6.2 \%$ and $7.8 \%$. The 10-year RSR was also observed the similar survival difference tendency (Fig. 3b, Supplementary Table S2).

Distributions of SES varied among different races. Most Whites were defined as low-poverty while most Blacks were defined as high- and medium-poverty (Supplementary Figure S1). In fact, the difference in survival between Whites and Blacks partially reflected the difference in survival between various SES subgroups. In the first decade, the median survival of Whites was 27 months higher than that of Blacks for 16 months, and the difference in survival in the fourth decade increased significantly to 18 months ( $p$ $<0.0001$; Fig. 4a). Similarly, compared with the high-poverty group, the survival advantage of the lowpoverty group was found, and the median survival gap kept widening and increased from 7 months to 12 months over time. In addition, Kaplan-Meier survival analysis showed significant differences among three SES subgroups in the past four decades (Fig. 4b).

\section{Discussion}

It was observed that the overall incidence of patients with epithelial ovarian cancer (EOC) declined from 11.4 to 9.0 per 100,000 in the four decades from 1974 to 2013. An improvement of EOC median survival rose from 27 months to 48 months, and 5-year RSR improved from 32.3-44.3\% in the same period. However, the median survival differences increased from 11 months to 18 months between Whites and Blacks and increased from 7 months to 12 months between low-poverty group and high-poverty group respectively over the past four decades.

It was showed that the incidence of EOC dropped by $21.0 \%$ from 11.4 to 9.0 per 100,000 in the past four decades. The trend of declining incidence was also found in all age groups. EOC patients had an improvement in median survival, and the 5-year RSR grew from $32.3-44.3 \%$ in the four decades. Despite absence of effective and specific screening methods in EOC, the improved medical care and the concept of early detection and early treatment of diseases widely spread among the population, and people are more actively involved in medical interaction, which greatly reduces the incidence of $\mathrm{EOC}^{2}$. More importantly, advances in modern imaging techniques, modified surgical methods, a combination of multiple chemotherapy and targeted antitumor schemes as well as individualized precise treatment mode, have greatly improved the survival of patients with EOC ${ }^{12-15}$.

In the four decades, despite Whites showed the highest incidence, the incidence gap become narrowing between Whites and Blacks over time as a result of a markedly rapid decline of incidence in Whites. Furthermore, it was demonstrated that Whites had an advanced survival, with higher 5-year and 10-year RSRs than Blacks, what's more important, the RSR gap grew widening between Whites and Blacks. 
Previous studies suggested that black patients with EOC tended to have a delayed treatment or be unlikely to receive standard-of-care treatment including chemotherapy and surgery ${ }^{16,17}$. That was closely related to the survival in EOC, which led to a poor prognosis in black patients ${ }^{18}$. Taken together, an urgent need required to improve the clinical treatment management and health care in black patients with EOC.

Furthermore, incidence of low-poverty group was the highest while that of high-poverty group was the lowest. Additionally, higher RSR was observed in the low-poverty group and the gap in survival between low- and high-poverty groups widened over time especially in the last decade. Prior studies indicated that SES was associated with cultural beliefs, insurance payer status and social support, which impacts the access to healthcare ${ }^{19,20}$. And high all-cause mortality closed correlated with less social support ${ }^{21,22}$. Moreover, the availability to better healthcare and medical consultation significantly affect treatment and survival of EOC ${ }^{19,23}$. When classified by SES, Blacks were found to be inferior to Whites in finance. Financial disadvantage is thought to keep blank patients away from medical counseling and treatment in the early stages of the disease. Therefore, the difference in SES between white and black patients with EOC may partly explain the changes in incidence and survival between them.

There were some limitations in this study. Although a large number of EOC patients were collected from the SEER program to analyze the incidence, survival and their tendencies over the past four decades, this study remained limited due to the result partly reflected the selected SEER sites and was not applicable to other geographic locations. Besides, the study might also be influenced by misclassification and variation

of SES within and among counties ${ }^{10,11}$. Furthermore, histological and therapeutic data were not analyzed in the study, which may affect the incidence and survival of EOC patients.

\section{Conclusions}

In conclusion, our study showed that the incidence and RSR of EOC patients had improved in the past four decades. But with passage of time, the survival gap between different races and SES gradually widened. What's more important, this study will promote the improvement of health care system and clinical management to erase the survival differences in SES groups and races identified in this study, and thereby to optimize the clinical outcome of patients with EOC.

\section{Abbreviations}

EOC

epithelial ovarian cancer

SEER

Surveillance, Epidemiology, and End Results

SES

socioeconomic status

RSR

relative survival rate 


\section{Declarations}

Acknowledgements

We would like to thank the database of Surveillance, Epidemiology, and End Results for providing an access for us to extract the data on patients with epithelial ovarian cancer.

\section{Author information}

\section{Affiliations}

Department of Gynecology, People's Hospital of Xinjiang Uygur Autonomous Region

Lili Han, Sulaiya Husaiyin, Miherinisha Maimaiti, Mayinuer Niyazi

Department of Gynecology, Affiliated Tumor Hospital, Xinjiang Medical University

Li Li

Correspondence to: Li Li, email: Lili89650@163.com, and Mayinuer Niyazi , email: Mayinuer1299@163.com

\section{Additional information}

\section{Competing interests}

The authors declare no competing interests.

\section{Author's Contributions}

Lili Han and Sulaiya Husaiyin wrote the main manuscript; Miherinisha Maimaiti prepared the table 1-2 and figure 1-4; Mayinuer Niyazi and Li Li extracted and analyzed the data from SEER program.

\section{Ethics approval and consent to participate}

Not applicable

Consent for publication

Not applicable

\section{Availability of data and materials}

The datasets generated and analyzed during the current study are available in the website [https://seer.cancer.gov/data/]

\section{Funding}




\section{References}

1. Siegel RL, Miller KD. Cancer statistics, 2019. 2019;69(1):7-34.

2. Chornokur G, Amankwah EK, Schildkraut JM, et al. Global ovarian cancer health disparities. Gynecologic oncology. 2013;129(1):258-64. Epub 2012/12/26.

3. Webb PM, Jordan SJ. Epidemiology of epithelial ovarian cancer. Best practice \& research Clinical obstetrics \& gynaecology. 2017;41:3-14. Epub 2016/10/17.

4. Towne SD, Jr. Socioeconomic, Geospatial, and Geopolitical Disparities in Access to Health Care in the US 2011-2015. International journal of environmental research and public health. 2017;14(6). Epub 2017/05/31.

5. Kendrick J, Nuccio E, Leiferman JA, et al. Primary Care Providers Perceptions of Racial/Ethnic and Socioeconomic Disparities in Hypertension Control. American Journal of Hypertension. 2015;28(9):1091-7.

6. Collins $\mathrm{Y}$, Holcomb K, Chapman-Davis E, et al. Gynecologic cancer disparities: a report from the Health Disparities Taskforce of the Society of Gynecologic Oncology. Gynecologic oncology. 2014;133(2):353-61. Epub 2014/01/11.

7. Organization. WH. International classification of diseases for oncology: Morphology. 3. Geneva: World Health Organization; 2000. .

8. Sun $\mathrm{H}, \mathrm{Ma} \mathrm{H}, \mathrm{Hong} \mathrm{G}$, et al. Survival improvement in patients with pancreatic cancer by decade: a period analysis of the SEER database, 1981-2010. Scientific reports. 2014;4:6747. Epub 2014/10/24.

9. Singh GK, et al. Area Socioeconomic Variations In US Cancer Incidence, Mortality, Stage, Treatment, And Survival, 1975-1999. National Cancer Institute, Bethesda Report No: NIH Publication No03-5417 (2003).

10. Krieger N, Chen JT, Waterman PD, et al. Geocoding and monitoring of US socioeconomic inequalities in mortality and cancer incidence: does the choice of area-based measure and geographic level matter?: the Public Health Disparities Geocoding Project. American journal of epidemiology. 2002;156(5):471-82. Epub 2002/08/28.

11. Krieger N, Chen JT, Waterman PD, et al. Race/ethnicity, gender, and monitoring socioeconomic gradients in health: a comparison of area-based socioeconomic measures--the public health disparities geocoding project. American journal of public health. 2003;93(10):1655-71. Epub 2003/10/10.

12. Kiselev VI, Ashrafyan LA, Muyzhnek EL, et al. A new promising way of maintenance therapy in advanced ovarian cancer: a comparative clinical study. BMC cancer. 2018;18(1):904. Epub 2018/09/22.

13. Bowtell DD, Bohm S, Ahmed AA, et al. Rethinking ovarian cancer II: reducing mortality from highgrade serous ovarian cancer. Nature reviews Cancer. 2015;15(11):668-79. Epub 2015/10/24. 
14. Harries M, Kaye SB. Recent advances in the treatment of epithelial ovarian cancer. Expert opinion on investigational drugs. 2001;10(9):1715-24. Epub 2002/01/05.

15. Katsumata N, Yasuda M, Isonishi S, et al. Long-term results of dose-dense paclitaxel and carboplatin versus conventional paclitaxel and carboplatin for treatment of advanced epithelial ovarian, fallopian tube, or primary peritoneal cancer (JGOG 3016): a randomised, controlled, open-label trial. The Lancet Oncology. 2013;14(10):1020-6. Epub 2013/08/21.

16. Dilley S, Erickson BK, Phillips $\mathrm{CE}$, et al. Do differences in medical comorbidities and treatment impact racial disparities in epithelial ovarian cancer? Gynecologic oncology. 2018;149(1):49-52. Epub 2018/04/02.

17. Barnholtz-Sloan JS, Schwartz AG, Qureshi F, et al. Ovarian cancer: changes in patterns at diagnosis and relative survival over the last three decades. American journal of obstetrics and gynecology. 2003;189(4):1120-7. Epub 2003/10/31.

18. Miller EM, Tymon-Rosario J, Strickler HD, et al. Racial Differences in Survival From Epithelial Ovarian Cancer Are Associated With Stage at Diagnosis and Use of Neoadjuvant Therapy: A 10-Year SingleInstitution Experience With a Racially Diverse Urban Population. International journal of gynecological cancer : official journal of the International Gynecological Cancer Society. 2018;28(4):749-56. Epub 2018/03/15.

19. Sakhuja S, Yun H, Pisu M, et al. Availability of healthcare resources and epithelial ovarian cancer stage of diagnosis and mortality among Blacks and Whites. 2017;10(1):57.

20. Samuel CA, Landrum MB, McNeil BJ, et al. Racial disparities in cancer care in the Veterans Affairs health care system and the role of site of care. American journal of public health. 2014;104 Suppl 4:S562-71. Epub 2014/08/08.

21. Idahl A, Hermansson A, Lalos A. Social support and ovarian cancer incidence - A Swedish prospective population-based study. Gynecologic oncology. 2018;149(2):324-8. Epub 2018/03/21.

22. Uchino BN. Social support and health: a review of physiological processes potentially underlying links to disease outcomes. Journal of behavioral medicine. 2006;29(4):377-87. Epub 2006/06/08.

23. Lee AW, Navajas EE, Liu L. Clear differences in ovarian cancer incidence and trends by ethnicity among Asian Americans. Cancer epidemiology. 2019;61:142-9. Epub 2019/06/30.

\section{Figures}


a

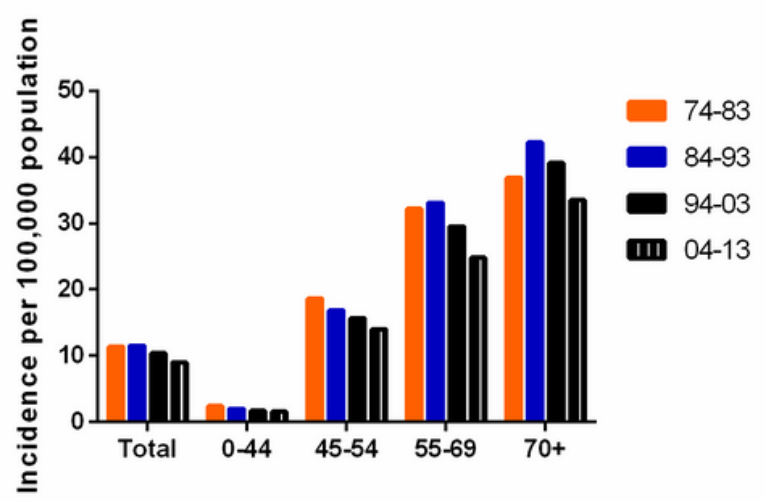

C

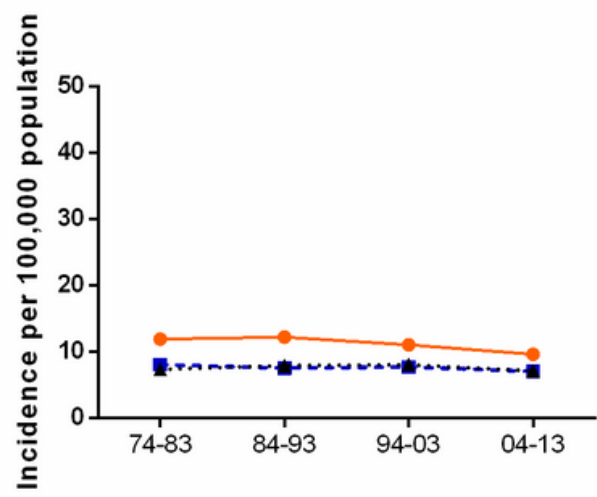

e

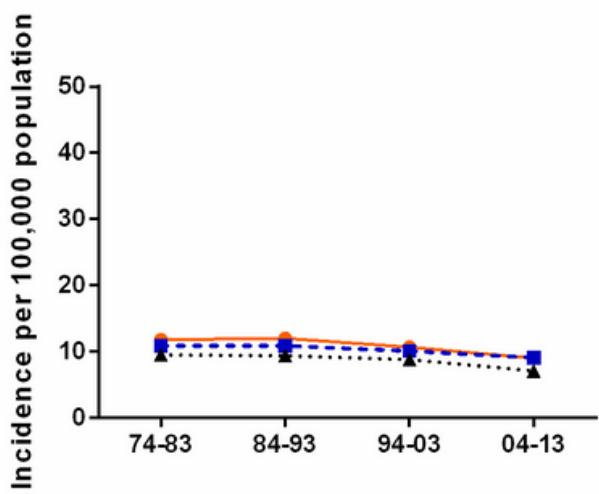

- White

-m. Black

... Other b

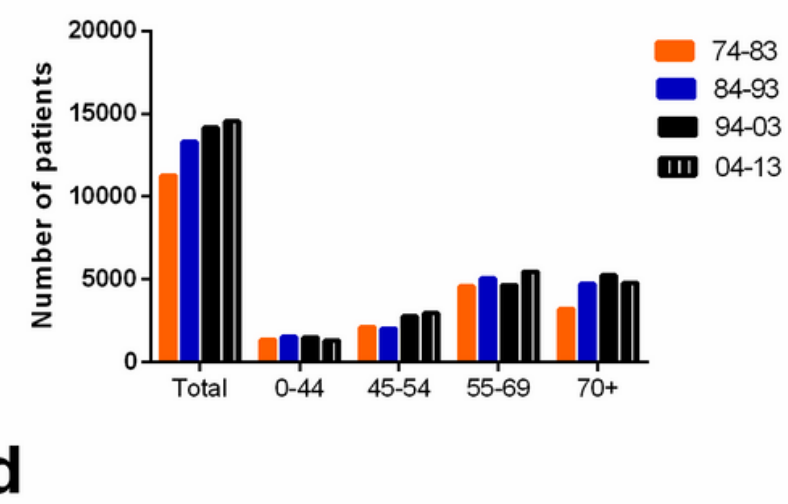

- Low Poverty

-m. Medium Poverty

.. High Poverty

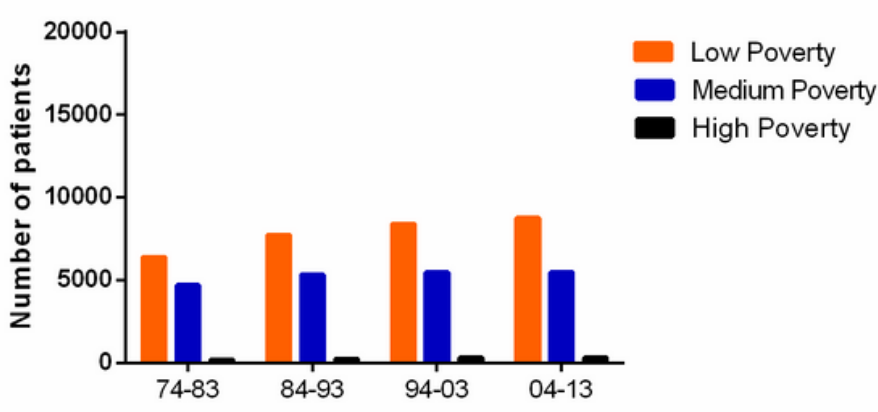

Figure 1

Summary incidences of patients diagnosed as epithelial ovarian cancer (EOC) from 1974 to 2013 at the original nine SEER sites. Incidence (a), and number (b) of EOC cases are shown by age group (total and ages 0-44, 45-54, 55-69, and 70+ years) and calendar period. Incidence (c,e) and number (d,f) of EOC cases are grouped by race and SES, respectively. 
a
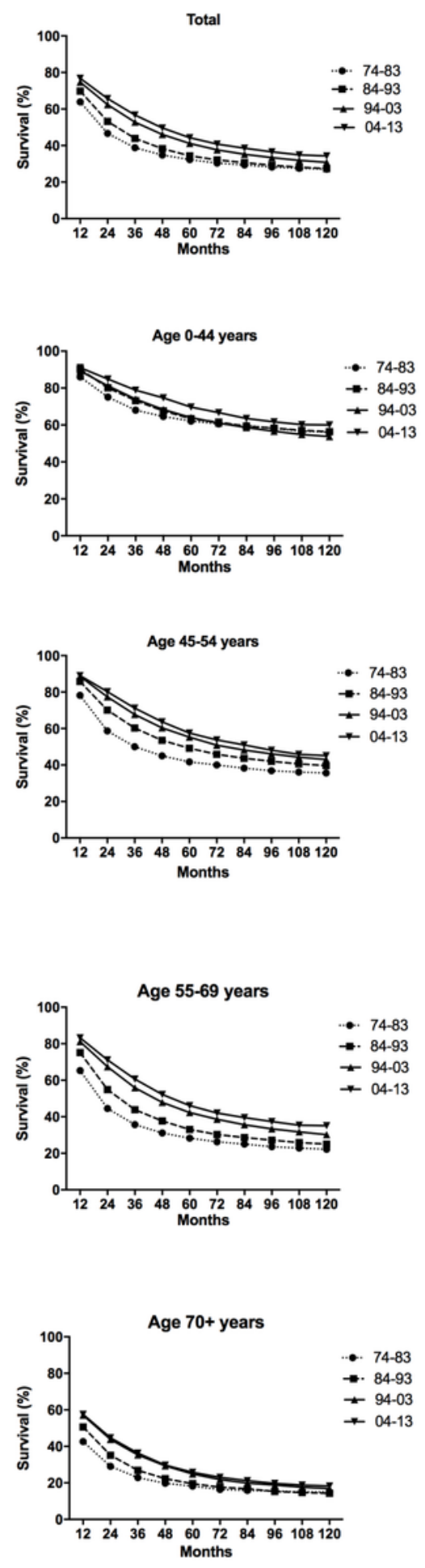

b
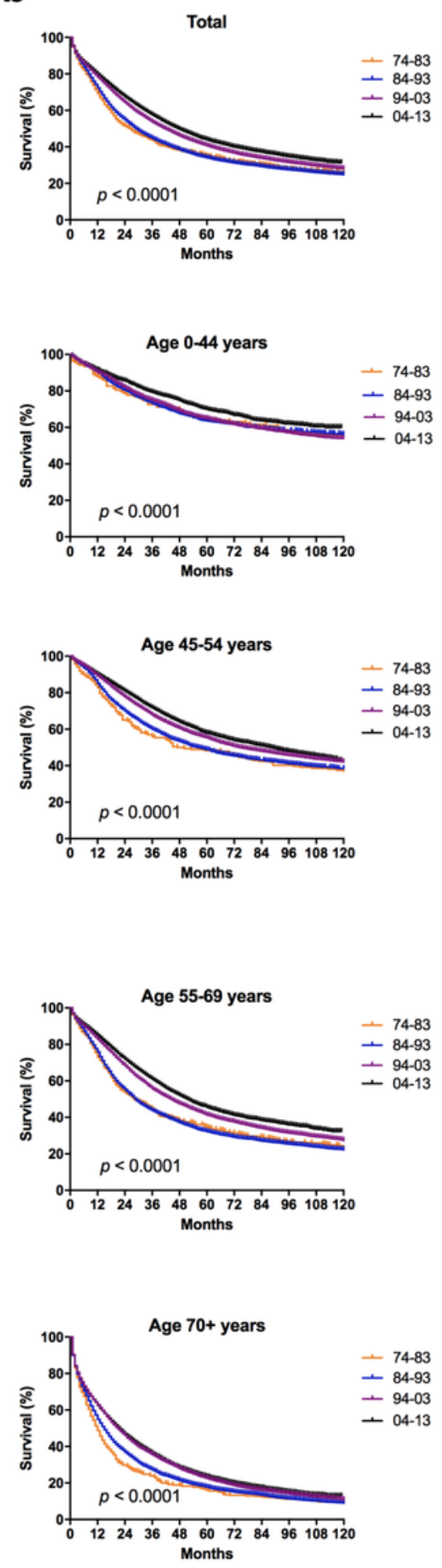

\section{Figure 2}

Trends in 10-year relative survival rates (a) and Kaplan-Meier survival analysis (b) for patients with EOC at 18 SEER sites in 1974-1983 (orange), 1984-1993 (blue), 1994-2003 (purple) and 2003-2012 (black) respectively according to age group (total and ages 0-44, 45-54, 55-69, and 70+ years) and calendar period. 
a

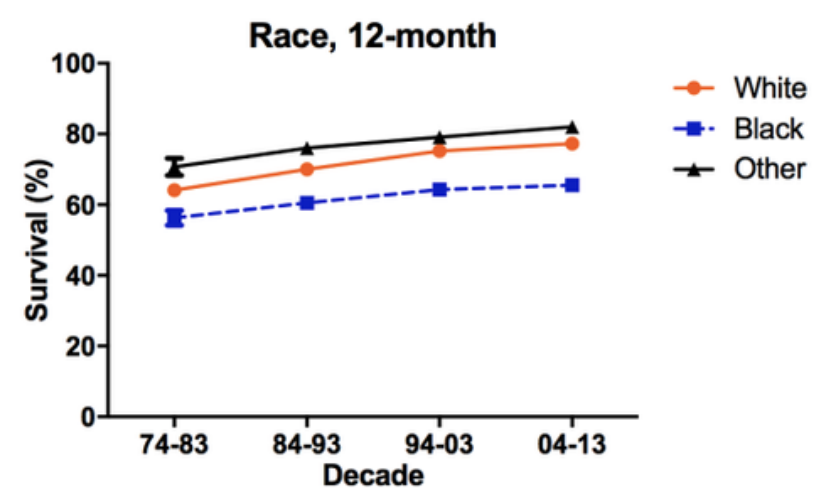

Race, 60-month

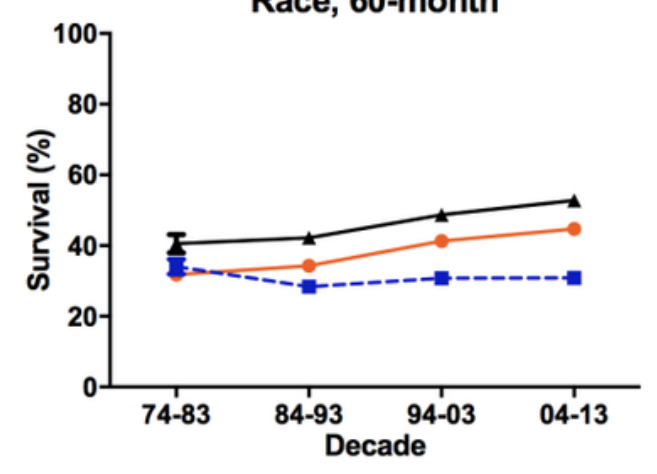

Race, 120-month

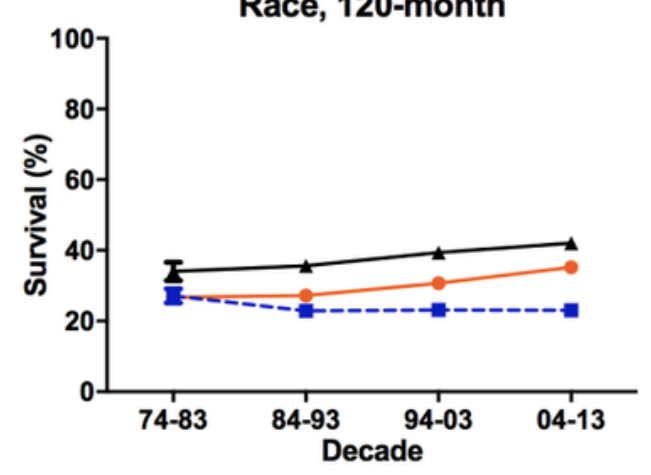

$\rightarrow$ White

$\rightarrow$ Black

- Other b

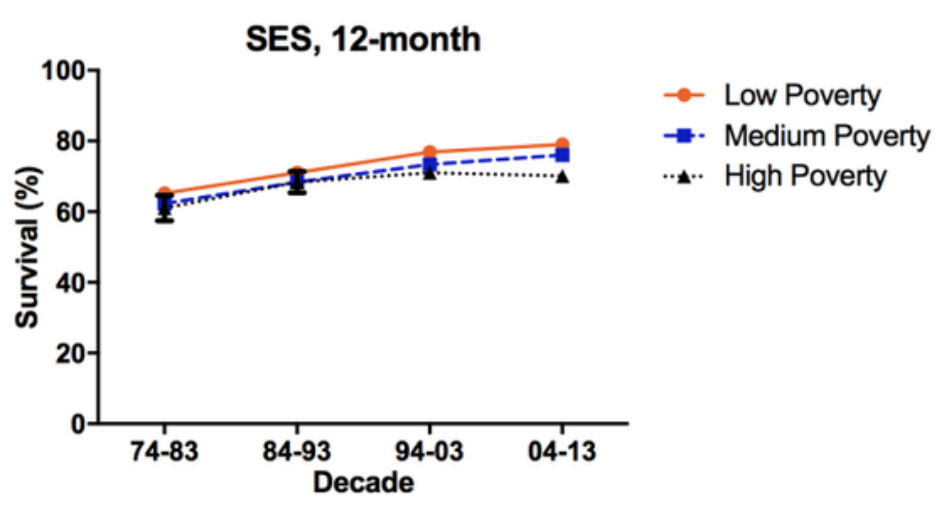

$\rightarrow$ White

$\rightarrow$ Black

- Other

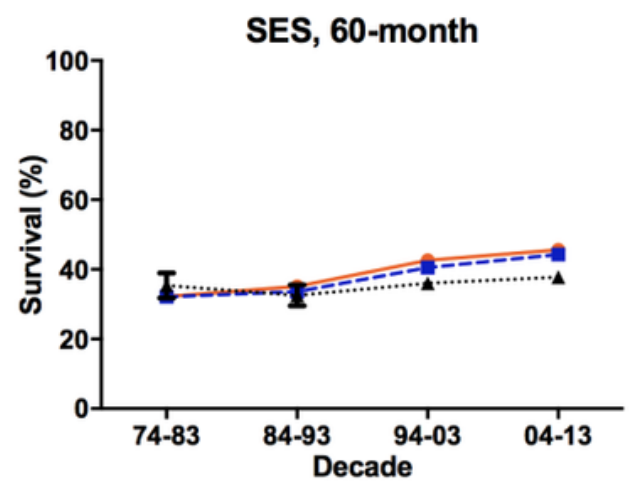

$\rightarrow$ Low Poverty

- Medium Poverty

.... High Poverty

\section{Figure 3}

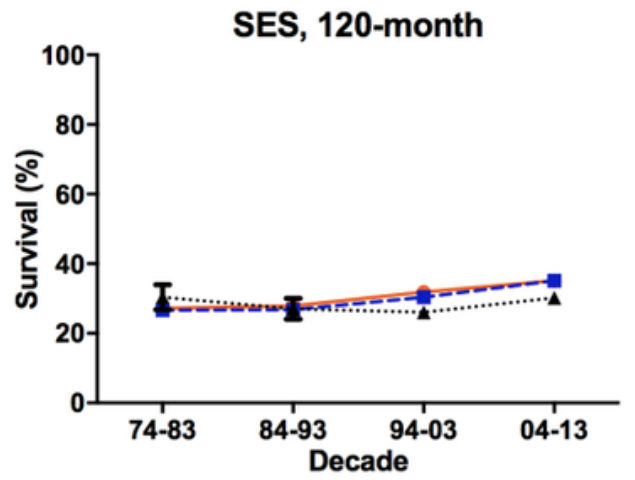

1-year, 5-year and 10-year relative survival rates (RSR) according to race (a) including White (orange), Black (blue) and Others (black), and SES/county-level poverty rates (b) in Low-poverty (orange), Mediumpoverty (blue) and High-poverty (black) for patients with EOC at 18 SEER sites from 1974 to 2013. 
a
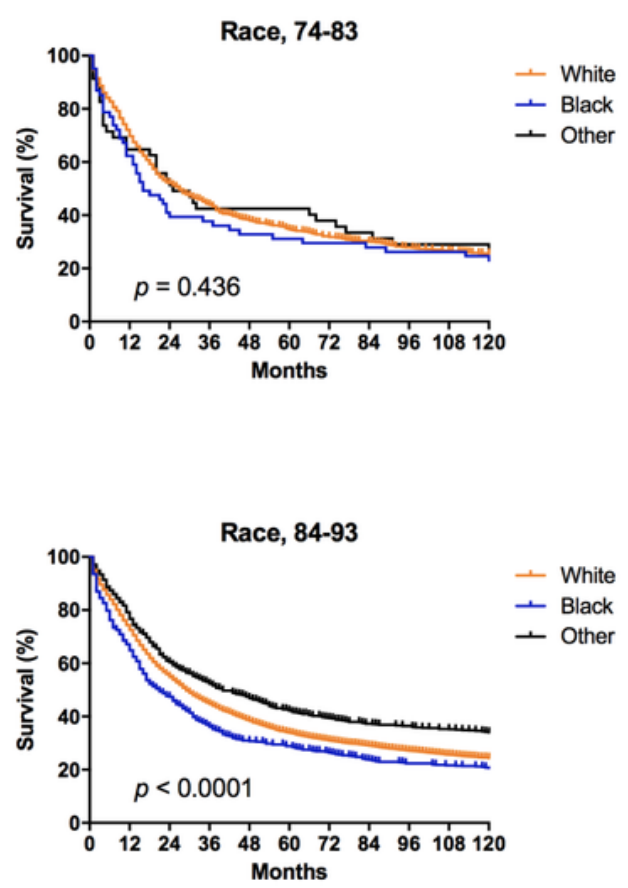

Race, 94-03

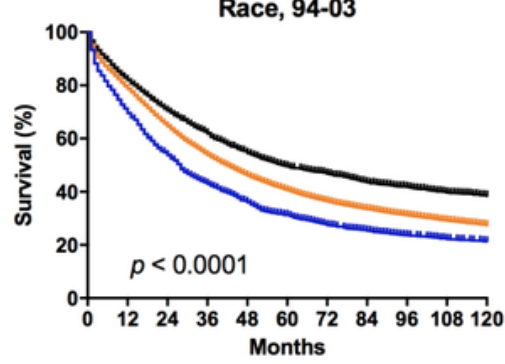

Race, 04-13

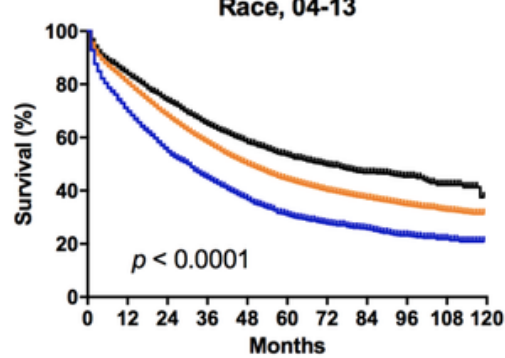

b

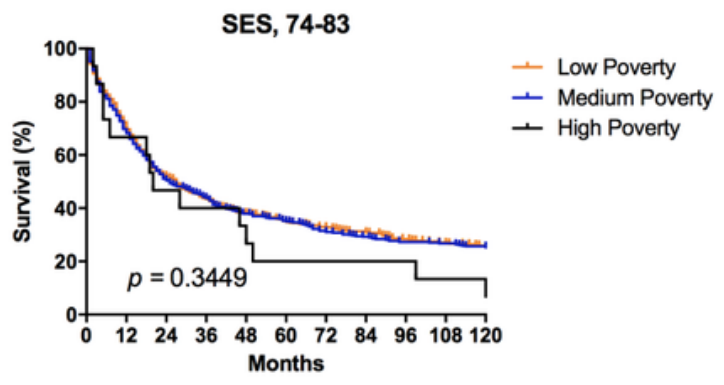

SES, 84-93

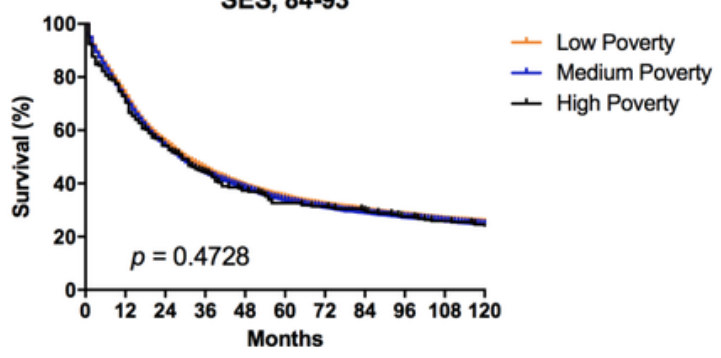

SES, 94-03

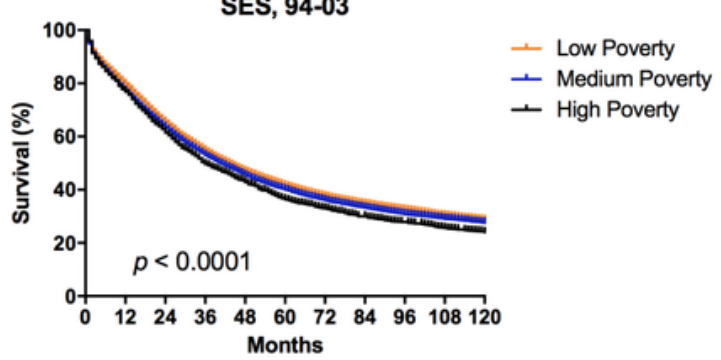

SES, 04-13

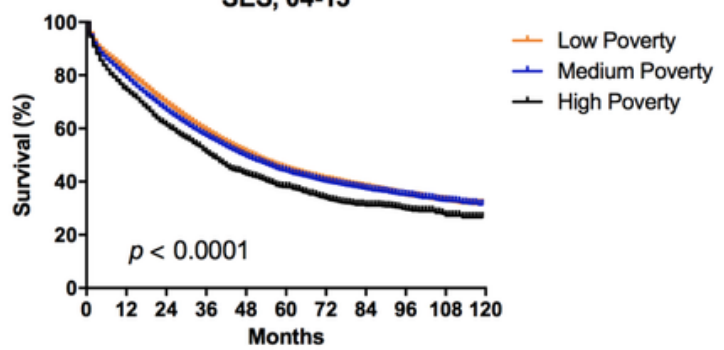

Figure 4

Kaplan-Meier survival analysis according to race (a) including White (orange), and Black (blue) and Others (black), and SES/ county-level poverty rates (b) in Low-poverty (orange), Medium-poverty (blue) and High-poverty (black) for patients with EOC at 18 SEER sites from 1974 to 2013. 
This is a list of supplementary files associated with this preprint. Click to download.

- SupplementaryFile.docx 